УДК $543,542.06$

\title{
ПРИМЕНЕНИЕ ПАРОФАЗНОГО АНАЛИЗА ДЛЯ ПОЛУЧЕНИЯ ОБЩЕГО ОБРАЗА ЛИСТЬЕВ ЭВКАЛИПТА ПРУТОВИДНОГО (ЕUCALYPTUS VIMINALIS LABILL)
}

(C) Л.В. Павлова ${ }^{*}$ И.А. Платонов, Н.В. Никитченко, И.Н. Колесниченко

\author{
Самарский национальный исследовательский университет имени академика \\ С.П. Королева, Московское шоссе, 34, Самара, 443086 (Россия), \\ e-mail: lora-pavlova@mail.ru
}

\begin{abstract}
Соотношение площадей пиков компонентов газового экстракта листьев эвкалипта прутовидного (Eucalyptus viminalis Labill): $\alpha$-пинена, камфена, $\beta$-пинена, $\alpha$-феландрена, п-цимена, 1,8-цинеола, 4-терпинеола, $\alpha$-терпинеола и аромадендрена приняли за характерный хроматографический спектр. Проведена оценка воспроизводимости хроматографических спектров листьев эвкалипта прутовидного, полученных методом ПФА-ГХ-МС и ПФА-ТФМЭ-ГХ-МС. Впервые предложено использовать полученные хроматографические спектры в качестве объективной химической идентификационной характеристики листьев эвкалипта прутовидного взамен ботаническим методам идентификации. Для оценки связи между образцами результаты обрабатывались методом главных компонент с использованием программы Statistica. Проведен анализ сорбционных свойств сорбентов Tenax ${ }^{\mathrm{TM}} \mathrm{TA}$, Carbopack B, Porapak Q, Haye Sep N, MN-202 по отношению к летучим органическим соединениям (ЛОС) листьев эвкалипта прутовидного. Предложено применение сорбционных трубок на основе инъекционных игл, заполненных выбранными полимерными сорбентами Porapak Q, Науе Sep N, MN-202, со сконцентрированными на них аналитами в качестве образцов состава ЛОС листьев эвкалипта прутовидного, в том числе и для идентификации данного растительного сырья. Установлены параметры сорбции ЛОС и сроки хранения сорбционных трубок с аналитами. Рекомендовано использовать полученные хроматографические спектры для создания базы данных характерных хроматографических спектров растительного сырья.

Ключевые слова: эвкалипт прутовидный, парофазный анализ, ГХ-МС анализ, хроматографический спектр, «фингерпринт», общий образ объекта, идентификация.
\end{abstract}

Работа поддержана Минобрнауки РФ в рамках государственного задания на выполнение работ, проект № 608 .

\section{Введение}

Одним из основных направлений развития современной аналитической химии является переход к многокомпонентному анализу, а также замене там, где это возможно, покомпонентного анализа распознаванием общего образа объекта [1]. Практическое приложение данные направления находят при идентификации растений по характерному хроматографическому профилю [2]. «Хроматографические профили, полученные посредством анализа газового экстракта лекарственных растений, продуктов жизнедеятельно-

Павлова Лариса Викторовна - кандидат химических наук, ведущий инженер кафедры химии, e-mail: lora-pavlova@mail.ru

Платонов Игорь Артемьевич - доктор технических наук, профессор, заведующий кафедрой химии, e-mail: lora-pavlova@mail.ru

Никитченко Наталья Викторовна - кандидат химических наук, ассистент кафедры химии, e-mail: lora-pavlova@mail.ru

Колесниченко Ирина Николаевна - кандидат химических наук, ассистент кафедры химии, e-mail: lora-pavlova@mail.ru сти животных и т.д. имеют настолько характерный вид, что могут служить «отпечатками пальцев» этих материалов даже без идентификации отдельных пиков» [2]. Понятие о характерном хроматографическом профиле, как о некой совокупности данных о количестве, интенсивности и характерном расположении пиков, которые принимаются за единую характеристическую особенность [2], позволяет нам рассматривать воспроизводимый хроматографический профиль исследуемого лекарственного расте-

\footnotetext{
* Автор, с которым следует вести переписку.
} 
ния как фактор оценки его подлинности и качества. Различие в терминологии обозначения метода распознавания общего образа объекта обусловлено приоритетом использования терминов в той или иной публикации. В зарубежных публикациях более привычен термин «фингерпринт» [3, 4], в отечественной литературе наряду с этим термином используется переводной вариант «отпечатки пальцев» либо хроматографический профиль [2]. Кроме этого в литературе встречается понятие «хроматографический спектр», введенное М.С. Вигдергаузом, которое означает совокупность хроматографических пиков, свойственных данному веществу [5]. Концепция хроматографического спектра и методы использования его для групповой, а в некоторых случаях и для индивидуальной идентификации неизвестных соединений широко применяется в нефтехимической промышленности [5-7].

Получение характерных хроматографических спектров рассматривается как один из этапов стандартизации растений и препаратов на их основе [3, 8-10]. Управление по продовольствию и медикаментам США предложило принять метод хроматографического «фингерпринта» для идентификации растительного сырья [4].

В последнее время ведется большое количество исследований по поиску оптимальных условий получения воспроизводимых «фингерпринт»-хроматограмм экстрактов лекарственных растений, как методом высокоэффективной жидкостной хроматографии [11-13], так и методом газовой хроматографии с масс-спектрометрическим детектированием (ГХ-МС) [14-17]. Однако в большинстве таких работ изучаются жидкие экстракты растений, при этом недостаточное внимание уделяется изучению характерных хроматографических спектров газовых экстрактов растений, полученных методом парофазного анализа (ПФА). Основным условием применения данного метода - наличие как самих летучих органических соединений (ЛОС) в газовом экстракте растений, так и достаточного числа компонентов для определения подлинности данного лекарственного растительного сырья [18]. Для повышения информативности ПФА, а также для увеличения числа идентификационных признаков, в ряде случаев рекомендуется применять твердофазную микроэкстракцию (ТФМЭ). Достоинствами этого метода экстракции являются быстрота, простота, отсутствие органических растворителей и минимизация влияния матрицы, а также снижение предела детектирования летучих органических соединений (ЛОС) до уровня ppt [19].

Целью нашего исследования было провести оценку возможности использования хроматографических спектров, полученных методом ПФА-ГХ-МС и ПФА-ТФМЭ-ГХ-МС в качестве идентификационной характеристики листьев эвкалипта прутовидного (Eucalyptus viminalis Labill), а также выполнить сравнение различных сорбентов для проведения ТФМЭ ЛОС листьев эвкалипта прутовидного.

\section{Экспериментальная часть}

Объекты исследования:

1) «Листья эвкалипта прутовидного (Eucalyptus viminalis Labill)» рода Эвкалипт (Eucalyptus), семейства Миртовые (Myrtaceae) ПКФ «Фитофарм» ООО;

2) «Листья эвкалипта прутовидного (Eucalyptus viminalis Labill)» рода Эвкалипт (Eucalyptus), семейства Миртовые (Myrtaceae)ООО «Красногорсклексредства»;

3) Листья эвкалипта шаровидного (Eucalyptus globulus) рода Эвкалипт (Eucalyptus), семейства Миртовые (Myrtaceae), предоставлены проф. В.А. Куркиным зав. каф. фармакогнозии с ботаникой Самарского государственного медицинского университета.

Листья эвкалипта прутовидного были выбраны для исследования в связи с отсутствием сведений о «фингерпринтах» в виде хроматографических спектров для данного растения.

Пробоподготовку листьев эвкалипта прутовидного, условия анализа, идентификацию ЛОС в газовом экстракте осуществляли, как описано в работе [20].

Для исследования были использованы сорбционные трубки, на основе инъекционных игл 0,8×38 мм [21]. Изготовление сорбционных трубок осуществлялось следующим образом: заточенная часть иглы срезается, а края завальцовываются внутрь. При этом длина трубки уменьшается и составляет 33 мм. Заполнение сорбционной трубки проводили подобно заполнению насадочной колонки. После чего для фиксации сорбента со стороны среза иглы вставлялась гранула MN-202 подходящего размера по принципу «шариковой ручки», со стороны «головки» иглы - кусочек силанизированного стекловолокна.

Для заполнения сорбционных трубок использовали сорбенты, приведенные в таблице 1. 
Таблица 1. Сорбенты, используемые в эксперименте, и их характеристики [22-24]

\begin{tabular}{|c|c|c|c|c|c|}
\hline Сорбент & $\begin{array}{c}\text { Максимальная } \\
\text { рабочая тем- } \\
\text { пература, }{ }^{\circ} \mathrm{C}\end{array}$ & $\begin{array}{c}\text { Удельная } \\
\text { поверх- } \\
\text { ность, } \mathrm{M}^{2} / \Gamma\end{array}$ & $\begin{array}{l}\text { Масса cop- } \\
\text { бента в } \\
\text { трубке, мг }\end{array}$ & $\begin{array}{c}\text { Размер } \\
\text { сорбента } \\
\text { меш }\end{array}$ & Примеры аналитов \\
\hline $\begin{array}{l}\text { Tenax }{ }^{\mathrm{TM}} \mathrm{TA} \\
\text { (полидифенил- } \\
\text { оксид) }\end{array}$ & 350 & 35 & 3 & $80 / 100$ & $\begin{array}{l}\text { Ароматические неполярные соединения } \\
\left.\text { (с температурой кипения более } 100^{\circ} \mathrm{C}\right) \text { и } \\
\text { менее летучие полярные соединения (с } \\
\left.\text { температурой кипения более } 150^{\circ} \mathrm{C}\right)\end{array}$ \\
\hline $\begin{array}{l}\text { Carbopack B } \\
\text { (графитированный } \\
\text { уголь) }\end{array}$ & $>400$ & 100 & 3 & $80 / 100$ & $\begin{array}{l}\text { Разнообразные ЛОС, включая кетоны, } \\
\text { спирты, альдегиды (с температурой ки- } \\
\left.\text { пения более } 75^{\circ} \mathrm{C}\right) \text { и все неполярные } \\
\text { соединения в указанном интервале лету- } \\
\text { чести и перфторуглеродные газы, при- } \\
\text { сутствующие в следовых количествах }\end{array}$ \\
\hline $\begin{array}{l}\text { Porapak Q } \\
\text { (этилвинилбензол/ } \\
\text { дивинилбензол) }\end{array}$ & 250 & 550 & 3 & $80 / 100$ & $\begin{array}{l}\text { Разнообразные ЛОС, включая кислород- } \\
\text { содержащие соединения (малополярный) }\end{array}$ \\
\hline $\begin{array}{l}\text { Науе Sep N } \\
\text { (дивинилбензол/ } \\
\text { этиленгликольди- } \\
\text { метакрилат) }\end{array}$ & 165 & 405 & 3 & $80 / 100$ & $\begin{array}{l}\text { Полярные соединения, галогены, серни- } \\
\text { стые соединения, спирты, альдегиды, } \\
\text { кетоны, гликоли, свободные жирные } \\
\text { кислоты, эфиры }\end{array}$ \\
\hline $\begin{array}{l}\text { MN-202 (сверхсши- } \\
\text { тый полистирол) }\end{array}$ & 200 & $\begin{array}{c}800-1000 \\
\text { бипористый }\end{array}$ & 1,7 & $40 / 60$ & Разнообразные соединения \\
\hline
\end{tabular}

Для проведения ТФМЭ сорбционную трубку вводили во флакон с растительным сырьем на глубину 1 см. Проточная ТФМЭ на сорбционную трубку осуществлялась со скоростью 2 см$^{3} /$ мин [21]. Термодесорбция проводилась в зависимости от максимальной рабочей температуры сорбента - $160-270{ }^{\circ} \mathrm{C}$. Объем вводимой пробы - $1 \mathrm{~cm}^{3}$.

Для классификации результатов по методу главных компонент использовалась программа Statistica 6.0.

\section{Обсуждение результатов}

При проведении ПФА листьев эвкалипта прутовидного визуально было отмечено, что хроматографический спектр ЛОС повторяется.

Для статистической обработки в качестве хроматографического спектра листьев эвкалипта прутовидного приняли соотношение площадей пиков компонентов, имеющих наиболее интенсивные пики на хроматограмме газового экстракта: $\alpha$-пинена, камфена, $\beta$-пинена, $\alpha$-феландрена, $n$-цимена, 1,8 -цинеола, 4-терпинеола, $\alpha$-терпинеола и аромадендрена (рис. 1).

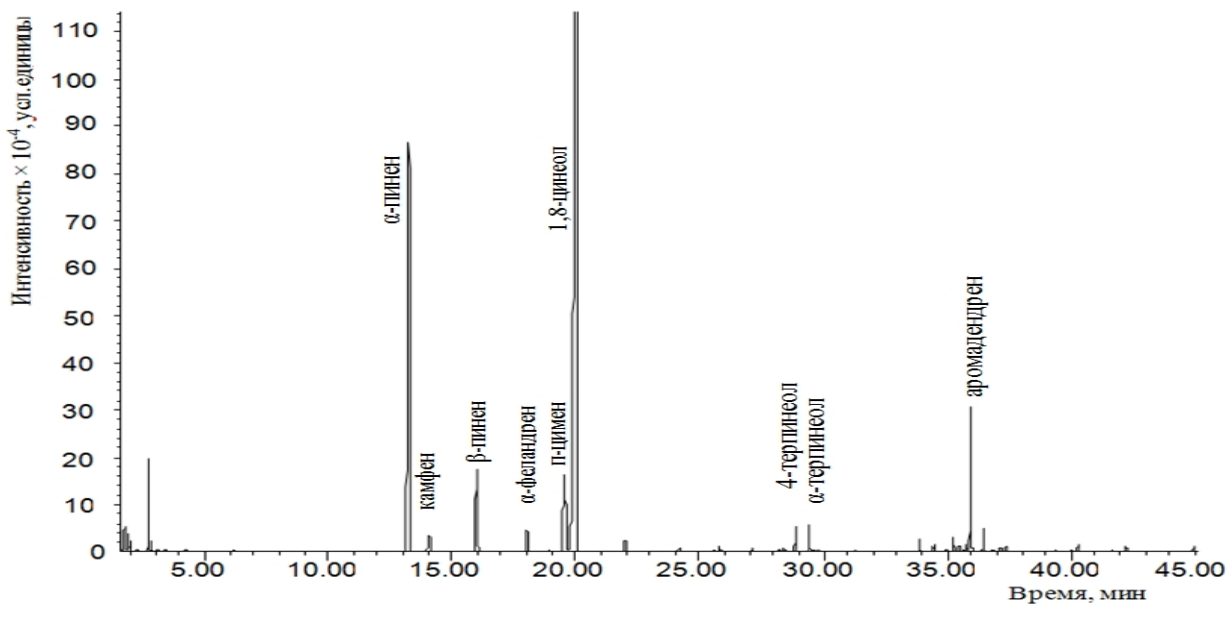

Рис. 1. Хроматографический спектр газового экстракта листьев эвкалипта прутовидного, полученный методом ПФА-ГХ-МС 
Для изучения возможности использования хроматографического спектра, полученного при ПФАГХ-МС листьев эвкалипта прутовидного, в качестве общего образа объекта, была проведена оценка хроматографического спектра на воспроизводимость. Кроме этого, проведено сравнение образцов листьев эвкалипта прутовидного разных производителей. Разброс значений в рамках одной партии сырья находится в пределах 1-20\% (табл. 2). По литературным данным ошибка при ПФА может достигать 50\% [2], поэтому можно сказать, что хроматографический спектр листьев эвкалипта прутовидного, полученный методом ПФА, является воспроизводимой характеристикой. Кроме того, сравнение образцов эвкалипта прутовидного производства ООО «Красногорсклексредства» (КЛС) и ПКФ «Фитофарм» (Фитофарм) показало, что соотношение компонентов воспроизводится с ОСКО 0,02-0,22 в зависимости от компонента.

Для оценки связи между образцами листьев эвкалипта прутовидного разных производителей результаты были обработаны методом главных компонент (рис. 2). Как следует из рисунка 2, данные образуют единую группу, следовательно, хроматографический спектр, полученный методом ПФА-ГХ-МС, может использоваться в качестве общего образа листьев эвкалипта прутовидного.

Таблица 2. Воспроизводимость хроматографического спектра листьев эвкалипта прутовидного, полученного методом ПФА

\begin{tabular}{|c|c|c|c|c|c|c|c|c|c|}
\hline \multirow[b]{2}{*}{ Образец } & \multicolumn{9}{|c|}{ Относительное содержание ЛОС, $A_{i}, \%$} \\
\hline & $\begin{array}{l}\text { 窇 } \\
\text { 焉 } \\
\frac{1}{d}\end{array}$ & 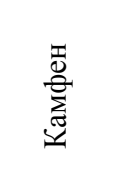 & 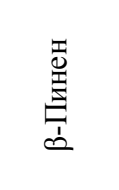 & 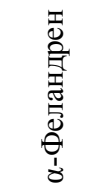 & 䓌 & 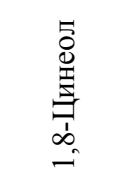 & 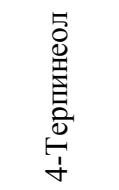 & 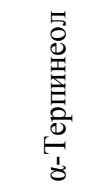 & 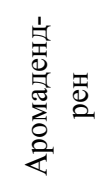 \\
\hline $\begin{array}{l}\text { КЛС } \bar{A}_{i} \pm \Delta \bar{A}_{\imath} \pm \Delta, \mathrm{n}=5, \\
\mathrm{P}=0,95\end{array}$ & $26,7 \pm 0,4$ & $1,0 \pm 0,1$ & $8,1 \pm 0,7$ & $1,5 \pm 0,5$ & $11,1 \pm 0,5$ & $47,1 \pm 1,2$ & $0,7 \pm 0,1$ & $0,7 \pm 0,2$ & $3,3 \pm 0,4$ \\
\hline $\mathrm{CKO}, S(A)$ & 0,3 & 0,1 & 0,6 & 0,4 & 0,4 & 1,0 & 0,1 & 0,1 & 0,3 \\
\hline ОСКО, $S_{r}(A)$ & 0,01 & 0,1 & 0,1 & 0,2 & 0,03 & 0,02 & 0,1 & 0,2 & 0,1 \\
\hline $\begin{array}{l}\text { Фитофарм, } \bar{A}_{i} \pm \Delta, \mathrm{n}=5, \\
\mathrm{P}=0,95\end{array}$ & $26,2 \pm 0,7$ & $1,2 \pm 0,2$ & $7,8 \pm 0,3$ & $1,4 \pm 0,4$ & $11,1 \pm 0,8$ & $47,2 \pm 0,3$ & $0,9 \pm 0,2$ & $0,9 \pm 0,1$ & $3,6 \pm 0,3$ \\
\hline $\mathrm{CKO}, S(A)$ & 0,6 & 0,2 & 0,3 & 0,3 & 0,7 & 0,3 & 0,2 & 0,1 & 0,3 \\
\hline OCKO, $S_{r}(A)$ & 0,02 & 0,1 & 0,03 & 0,2 & 0,06 & 0,01 & 0,2 & 0,1 & 0,07 \\
\hline$\mu \pm \Delta, n=10, P=0,95$ & $26,4 \pm 0,4$ & $1,1 \pm 0,1$ & $7,9 \pm 0,3$ & $1,4 \pm 0,2$ & $11,1 \pm 0,4$ & $47,1 \pm 0,5$ & $0,8 \pm 0,1$ & $0,8 \pm 0,1$ & $3,4 \pm 0,2$ \\
\hline СКО, $\sigma(A)$ & 0,5 & 0,2 & 0,4 & $\mathbf{0 , 3}$ & 0,5 & $\mathbf{0 , 7}$ & 0,2 & 0,1 & 0,3 \\
\hline ОСКО, $\sigma_{r}(A)$ & $\mathbf{0 , 0 2}$ & 0,1 & 0,06 & 0,2 & 0,05 & $\mathbf{0 , 0 1}$ & 0,2 & 0,1 & 0,1 \\
\hline
\end{tabular}

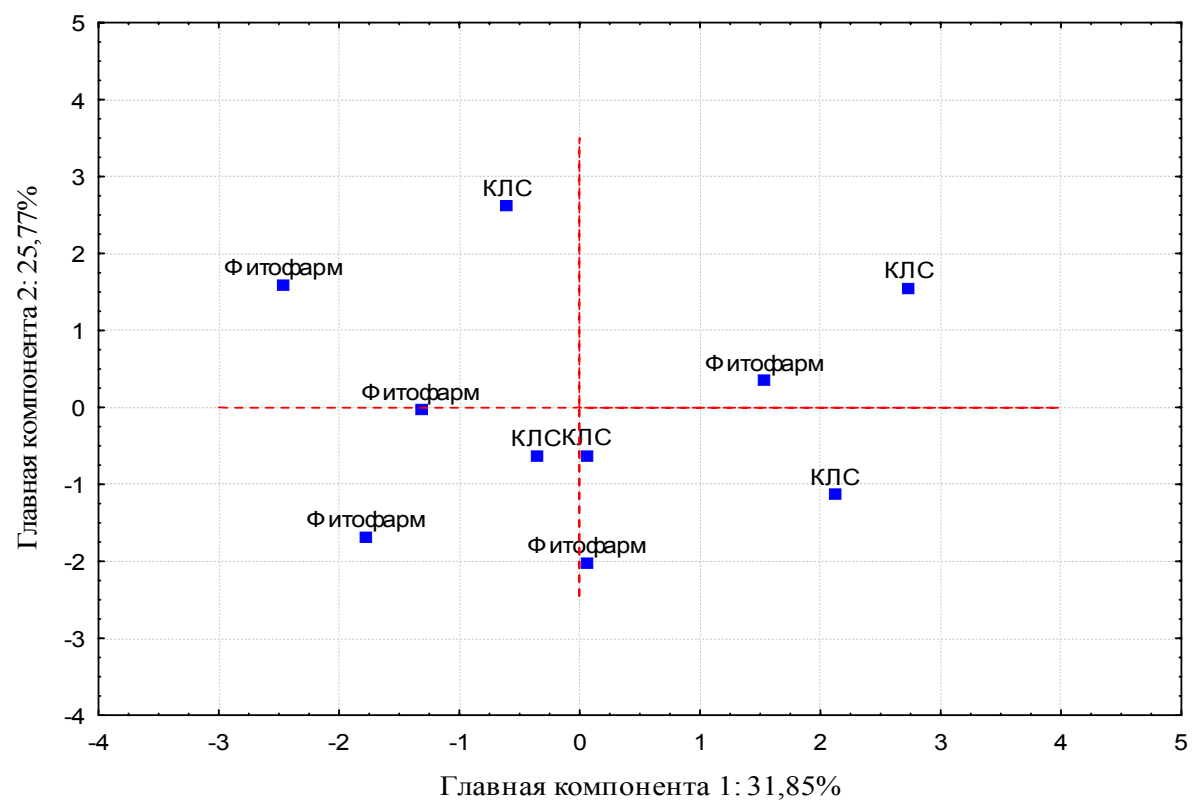

Рис. 2. График образцов эвкалипта прутовидного в координатах главных компонент (КЛС - ООО «Красногорсклексредства», Фитофарм - ПКФ «Фитофарм» ООО) 
В настоящее время идентификация растений производится только ботаническими методами $[25,26]$, которые основаны на знании особенностей строения растений. Использование химических методов идентификации позволит исключить субъективный фактор. Рассматриваемый в данном исследовании метод идентификации по характерному хроматографическому спектру уже находит применение в китайской традиционной медицине, когда наблюдается существенное морфологическое сходство между растениями [27]. Для оценки возможности идентификации листьев эвкалипта прутовидного по хроматографическому спектру, полученному методом ПФА-ГХ-МС, было проведено сравнение с хроматографическим спектром листьев эвкалипта шаровидного (рис. 3). Хроматографический спектр в виде соотношения площадей пиков компонентов эфирного масла эвкалипта шаровидного рассматривался в публикации [28]. Для проведения сравнения за хроматографический спектр листьев эвкалипта шаровидного приняли соотношения тех же компонентов, которые образуют хроматографический спектр листьев эвкалипта прутовидного. Компонент со временем удерживания 32,3 мин, идентифицированный как эндоборнилацетат при ГХ-МС анализе листьев эвкалипта шаровидного, не учитывался при расчете процентного соотношения компонентов, поскольку не обнаружен в газовом экстракте листьев эвкалипта прутовидного.

Обработка результатов методом главных компонент (МГК) продемонстрировала четкую группировку данных по видам эвкалипта (рис. 4).

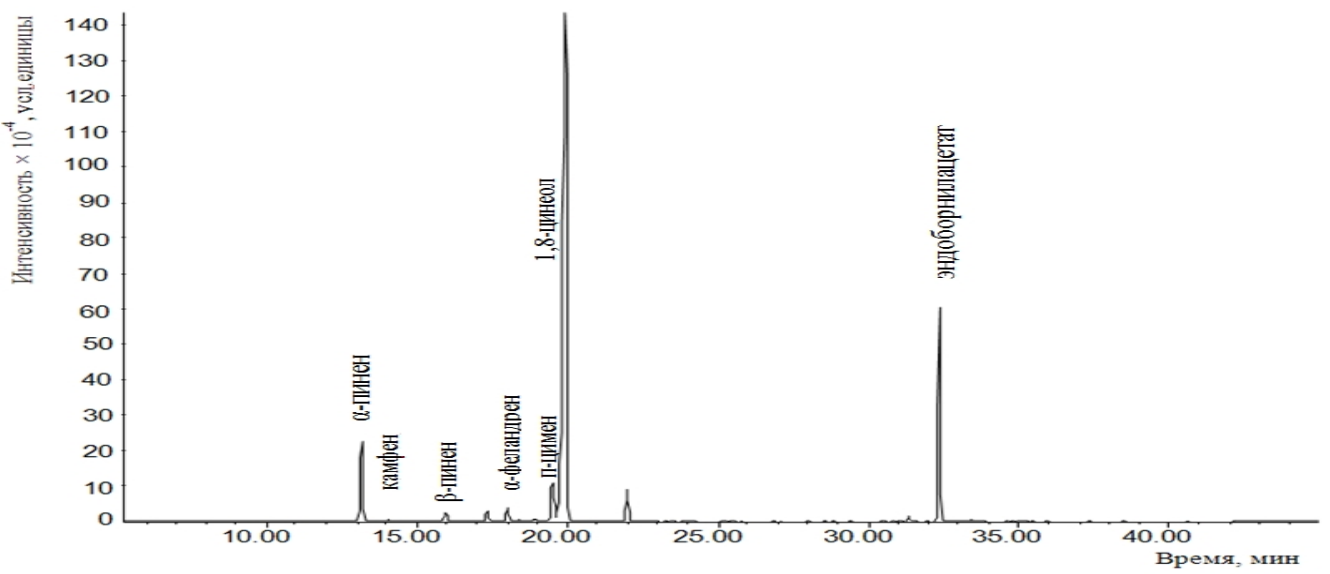

Рис. 3. Хроматографический спектр листьев эвкалипта шаровидного, полученный методом ПФА-ГХ-МС

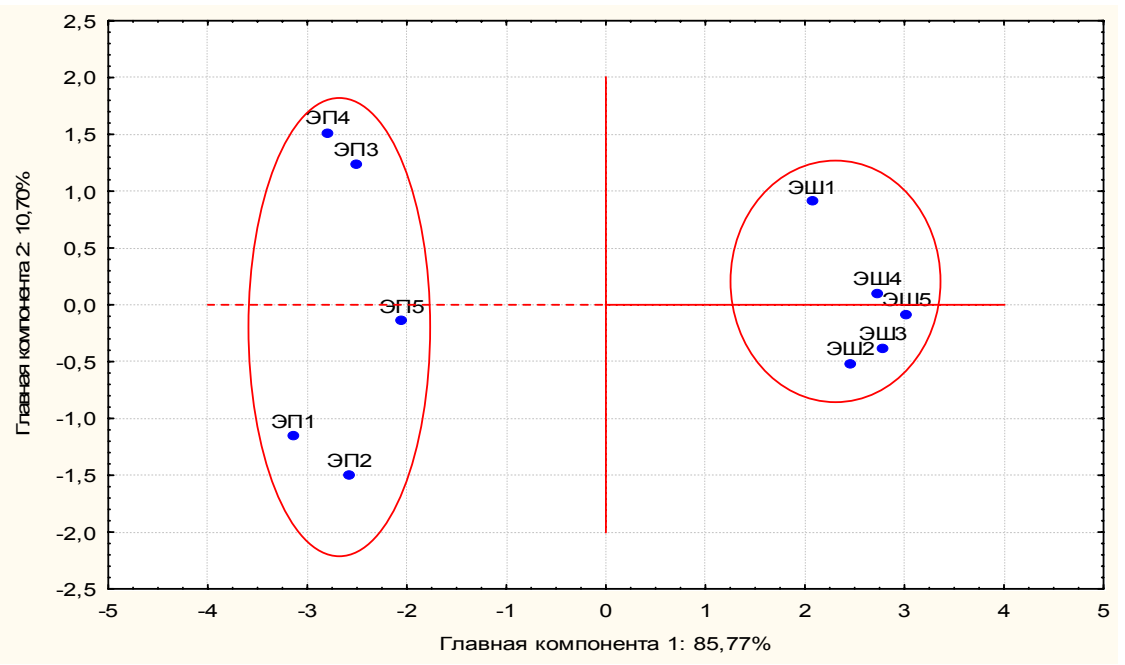

Рис. 4. График результатов ПФА-ГХ-МС листьев эвкалипта прутовидного (ЭП) и листьев эвкалипта шаровидного (ЭШ) в координатах главных компонент 1 и 2 
Из полученных данных следует, что хроматографический спектр листьев эвкалипта прутовидного, полученный методом ПФА-ГХ-МС, является воспроизводимой характеристикой и может использоваться для идентификации данного вида эвкалипта.

Идентификация растения по характерному хроматографическому спектру, полученному методом ПФА-ГХ-МС, может осуществляться при наличии базы данных хроматографических спектров либо при наличии эталонного растения. Мы предлагаем зафиксировать хроматографический спектр ЛОС листьев эвкалипта прутовидного на сорбционных микротрубках и в дальнейшем применять их в качестве образцов состава по принципу парофазных источников газовых смесей (ПИГС) [29], когда целевой компонент зафиксирован на сорбенте. Отличие предлагаемых образцов состава ЛОС растений от ПИГС состоит в том, что десорбция целевых компонентов происходит практически мгновенно при помещении сорбционных микротрубок в испаритель хроматографа, и в результате ГХ-МС анализа получаем хроматографический спектр искомого растения.

Для изготовления предлагаемых образцов состава в первую очередь необходимо подобрать сорбент, который отвечает следующим требованиям: хроматографический спектр, полученный после десорбции с сорбента, должен совпадать с хроматографическим спектром растения, полученным при ПФА-ГХ-МС, а также сорбент должен удерживать аналиты в необходимом соотношении в течение продолжительного времени для хранения сорбционных трубок со сконцентрированными на них ЛОС. Для достижения этой цели были поставлены следующие задачи.

1. Определение объема до проскока для выбранных сорбентов.

2. Определение оптимального объема газового экстракта для концентрирования на сорбционные трубки с последующей термодесорбцией.

3. Определение срока хранения сорбционных микротрубок со сконцентрированными на них аналитами.

Известно, что определение объема до проскока осуществляется до выхода первого компонента смеси [24]. Оценка сорбционных свойств указанных выше сорбентов проводилась для листьев эвкалипта прутовидного - по $\alpha$-пинену, компоненту, который элюируется первым на хроматограмме газового экстракта, а также по 1,8-цинеолу, который имеет наиболее интенсивный пик на хроматограмме.

Результаты определения объема до проскока приведены в таблице 3. Следует отметить особые условия концентрирования ЛОС растительного сырья: пробоотбор происходит фактически при $100{ }^{\circ} \mathrm{C}$. С повышением температуры насыщения уменьшается сорбционная емкость сорбента [22]. К тому же объем до проскока для пористых полимеров уменьшается в два раза при повышении температуры на $10^{\circ} \mathrm{C}$ [24]. Объемы «до проскока» для углеродного молекулярного сита в меньшей степени зависят от температуры и расхода, однако они существенно уменьшаются при высоком содержании летучих органических паров или высокой относительной влажности [24].

Из представленной таблицы следует, что MN 202 оказался самым эффективным сорбентом по отношению к $\alpha$-пинену, применительно к другим компонентам - Porapak Q. Carbopack B слабо удерживает целевые компоненты, а Тенакс ТА совсем не удерживает $\alpha$-пинен и 1,8 -цинеол. Газовый экстракт эвкалипта прутовидного проходит через сорбционную трубку, заполненную Тенаксом ТА практически без изменений концентрации.

Выходные кривые подтверждают хорошие сорбционные свойства MN 202, Haye Sep N, Porapak Q (рис. 5). Для Porapak Q кривая имеет более пологий вид, при этом концентрация компонентов в газовом экстракте, пропущенном через сорбент, так и не достигает их концентрации в исходном газовом экстракте, при этом объем газового экстракта эвкалипта прутовидного, пропущенного через сорбент, составил 300 мл. Это происходит за счет недостаточной элюирующей силы подвижной фазы. Для данного сорбента для достижения выходной кривой значения $\mathrm{C} / \mathrm{C}_{\mathrm{cp}}=1$ необходимо проводить ТФМЭ с большей скоростью.

Таблица 3. Объем «до проскока» для $\alpha$-пинена и 1,8 -цинеола

\begin{tabular}{l|c|c}
\hline \multirow{2}{*}{\multicolumn{1}{c|}{ Сорбент }} & \multicolumn{2}{|c}{ «Объем до проскока, см ${ }^{3}$} \\
\cline { 2 - 3 } & $\alpha$-пинен & 1,8 -цинеол \\
\hline Tenax TM TA & 0 & 0 \\
Carbopack B & 3 & 30 \\
Porapak Q & 12 & 14 \\
Haye Sep N & 6 & 14 \\
MN-202 & 13 & \\
\hline
\end{tabular}




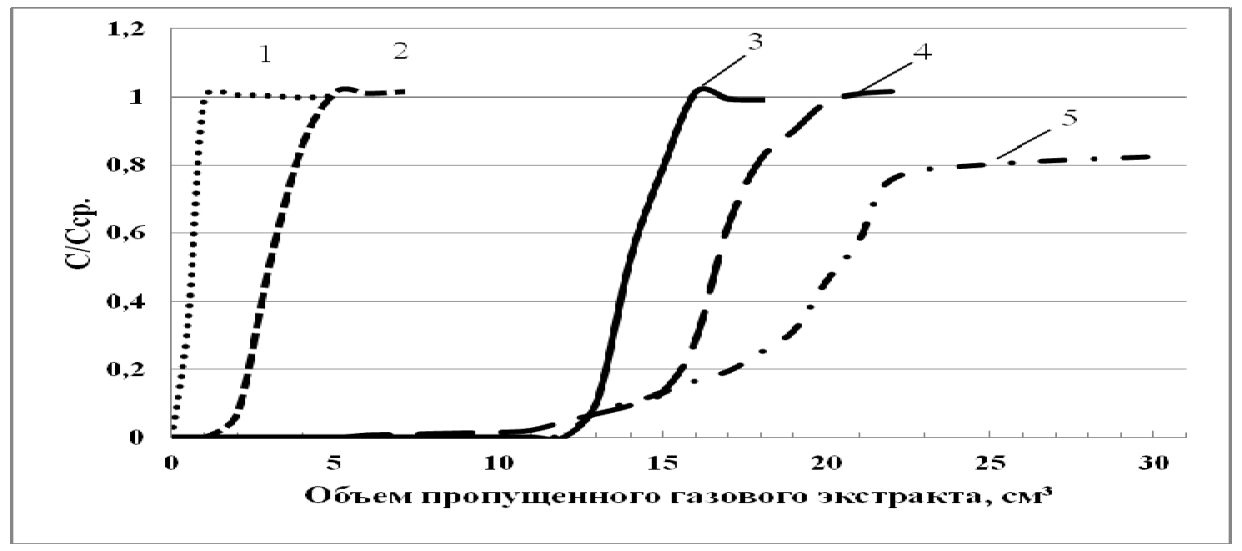

Рис. 5. Выходные кривые при концентрировании ЛОС эвкалипта прутовидного: 1 - Теnax ТА, 2 - Carbopak B, 3 - MN-202, 4 - HayeSep N, 5 - Porapack Q

Как известно, концентрирование летучих органических соединений из воздуха производится в объеме, находящемся в диапазоне объема «до проскока» первого компонента смеси [24]. Исходя из полученных результатов, объем газового экстракта эвкалипта прутовидного для проведения ТФМЭ на сорбционную трубку, заполненную Carbopack B, должен находится в диапазоне до $3 \mathrm{~cm}^{3}$, заполненную MN $202-$ до $13 \mathrm{~cm}^{3}$, Науе Sep N - до $6 \mathrm{~cm}^{3}$, Porapak Q - до $12 \mathrm{~cm}^{3}$.

Для выбора оптимального объема концентрирования газового экстракта исходили из следующих ограничений: минимальный расход растительного сырья, воспроизводимость хроматографического спектра. Было установлено, что для листьев эвкалипта прутовидного концентрирование пробы из одного флакона целесообразно проводить в объеме $3 \mathrm{~cm}^{3}$, поскольку такой объем газового экстракта образуется во флаконе для ПФА. Увеличение объема концентрирования газового экстракта из одного флакона практически не приводит к увеличению площадей пиков.

Для увеличения степени извлечения компонентов газового экстракта необходимо после каждых $3 \mathrm{~cm}^{3}$ менять флакон с пробой. В нашем случае концентрирование 3 см ${ }^{3}$ газового экстракта позволяет сэкономить время анализа и растительное сырье, предоставляя при этом воспроизводимые результаты (табл. 4).

Таблица 4. Характеристики воспроизводимости соотношения компонентов при ТФМЭ газового экстракта эвкалипта прутовидного в объеме $3 \mathrm{~cm}^{3}$

\begin{tabular}{|c|c|c|c|c|c|c|c|c|c|}
\hline \multirow[b]{2}{*}{ Сорбент } & \multicolumn{9}{|c|}{ Относительное содержание ЛОС, $A_{i}, \%$} \\
\hline & 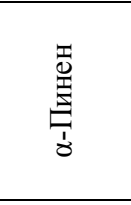 & 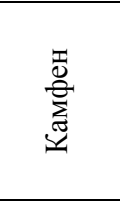 & 志 & 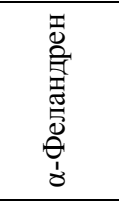 & 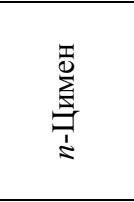 & 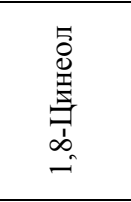 & 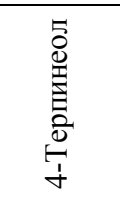 & 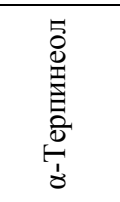 & 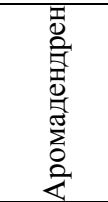 \\
\hline $\begin{array}{l}\text { Tenax TA, } \bar{A}_{i} \pm \Delta, \mathrm{n}=5 \\
\mathrm{P}=0,95\end{array}$ & $6,6 \pm 0,5$ & $0,5 \pm 0,4$ & $2,5 \pm 0,4$ & $3,6 \pm 0,5$ & $36,9 \pm 0,4$ & $35,3 \pm 0,8$ & $5,7 \pm 0,5$ & $5,2 \pm 0,9$ & $3,8 \pm 0,5$ \\
\hline OCKO, $S_{r}(A)$ & 0,06 & 0,6 & 0,1 & 0,1 & 0,01 & 0,01 & 0,07 & 0,1 & 0,1 \\
\hline $\begin{array}{l}\text { Porapak } \mathrm{Q}, \bar{A}_{i} \pm \Delta, \mathrm{n}=5, \\
\mathrm{P}=0,95\end{array}$ & $21,6 \pm 0,5$ & $1,1 \pm 0,1$ & $6,4 \pm 0,2$ & $1,4 \pm 0,5$ & $8,7 \pm 0,4$ & $52,2 \pm 0,3$ & $1,3 \pm 0,5$ & $1,5 \pm 0,4$ & $5,7 \pm 0,5$ \\
\hline OCKO, $S_{r}(A)$ & 0,02 & 0,07 & 0,02 & 0,3 & 0,03 & 0,01 & 0,3 & 0,2 & 0,1 \\
\hline $\begin{array}{l}\text { Carbopak B, } \bar{A}_{i} \pm \Delta, \mathrm{n}=5, \\
\mathrm{P}=0,95\end{array}$ & $9,0 \pm 0,2$ & $1,8 \pm 0,1$ & $2,4 \pm 0,3$ & $1,9 \pm 0,3$ & $15,2 \pm 0,5$ & $56,6 \pm 0,7$ & $2,4 \pm 0,3$ & $2,4 \pm 0,3$ & $8,4 \pm 0,4$ \\
\hline OCKO, $S_{r}(A)$ & 0,02 & 0,05 & 0,08 & 0,1 & 0,02 & 0,01 & 0,08 & 0,1 & 0,04 \\
\hline $\mathrm{MN}-202, \bar{A}_{i} \pm \Delta, \mathrm{n}=5, \mathrm{P}=0,95$ & $23,1 \pm 0,4$ & $1,2 \pm 0,2$ & $5,5 \pm 0,5$ & $1,3 \pm 0,2$ & $10,2 \pm 0,2$ & $54,3 \pm 0,6$ & $1,0 \pm 0,1$ & $1,0 \pm 0,2$ & $2,4 \pm 0,4$ \\
\hline OCKO, $S_{r}(A)$ & 0,01 & 0,1 & 0,08 & 0,1 & 0,02 & 0,01 & 0,1 & 0,1 & 0,1 \\
\hline $\begin{array}{l}\text { Haye Sep N, } \bar{A}_{i} \pm \Delta, \mathrm{n}=5, \\
\mathrm{P}=0,95\end{array}$ & $17,0 \pm 0,5$ & $1,1 \pm 0,1$ & $5,5 \pm 0,3$ & $1,7 \pm 0,2$ & $10,2 \pm 0,1$ & $57,8 \pm 0,4$ & $1,2 \pm 0,1$ & $1,3 \pm 0,1$ & $4,2 \pm 0,2$ \\
\hline $\mathrm{OCKO}, S_{r}(A)$ & 0,02 & 0,1 & 0,04 & 0,1 & 0,01 & 0,01 & 0,1 & 0,1 & 0,03 \\
\hline
\end{tabular}


На рисунке 6 представлены хроматографические спектры, полученные при ПФА-ТФМЭ-ГХ-МС анализе при концентрировании 3 см$^{3}$ экстракта. Термодесорбция со всех сорбентов, за исключением Тепах TA, демонстрирует визуальное совпадение хроматографических спектров с хроматографическим спектром, полученным методом ПФА-ГХ-МС. В то же время интенсивность пиков на полученных хроматограммах позволяет провести необходимые расчеты концентрации и успешно идентифицировать компоненты.
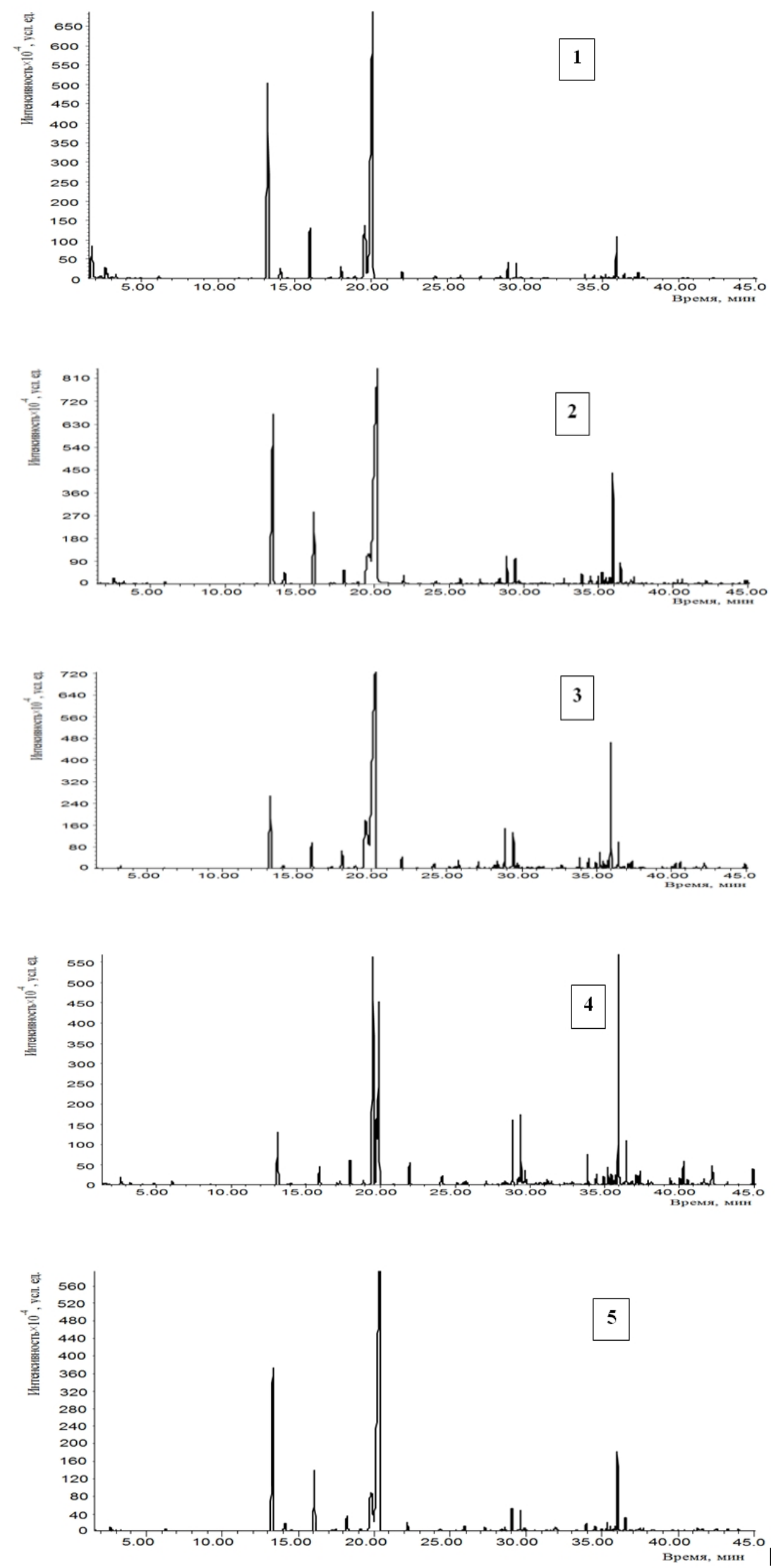

Рис. 6. Хроматограммы, полученные при ПФАТФМЭ-ГХ-МС 3 мл газового экстракта листьев эвкалипта прутовидного: 1) MN 202, 2) Porapak Q, 3) Carbopak B, 4) Tanax TA, 5) Haye Sep $N$ 
Для оценки возможности использования сорбционных микротрубок со сконцентрированными на них аналитами в качестве образцов состава ЛОС листьев эвкалипта прутовидного, необходимо оценить совпадение хроматографических спектров, полученных методами ПФА-ГХ-МС и ПФА-ТФМЭ-ГХ-МС. Для этого результаты были обработаны методом главных компонент, где в качестве переменных использовали соотношение площадей хроматографических пиков компонентов, указанных выше (рис. 7). Данные разделились на три кластера. Первый включает в себя наиболее обширную группу данных - ПФА- ГХ-МС листьев эвкалипта прутовидного, ПФА-ТФМЭ-ГХ-МС с использованием сорбционных микротрубок, заполненных MN 202, Porapak Q и Науе Sep N, следовательно, данные сорбенты могут быть взаимозаменяемы при использовании в сорбционных микротрубках. Кроме того, сорбционные микротрубки, заполненные данными сорбентами, могут использоваться для получения общего образа листьев эвкалипта прутовидного. На рисунке 7 выделяются две группы результатов ПФА-ТФМЭ-ГХ-МС на Carbopack В и Теnaх ТА, гипотетически их использование возможно для ТФМЭ ЛОС эвкалипта прутовидного, но они не обладают универсальностью MN 202, Porapak Q и Наye Sep N, к тому же слабо удерживают легколетучие компоненты газового экстракта.

Для изучения возможности идентификации эвкалипта прутовидного по хроматографическим спектрам при обработке данных МГК были добавлены результаты ПФА-ГХ-МС листьев эвкалипта шаровидного (рис. 8). Полученные результаты демонстрируют четкое выделение группы данных ПФА листьев эвкалипта шаровидного, доказывая при этом возможность использования хроматографического спектра, полученного ПФА-ГХ-МС и ПФА-ТФМЭ-ГХ-MC на MN 202, Porapak Q и HayeSep N, в качестве общего образа объекта для идентификации листьев эвкалипта прутовидного.

Одним из главных параметров оценки образцов состава ЛОС листьев эвкалипта прутовидного на основе сорбционных микротрубок является срок хранения сорбционных микротрубок со сконцентрированными на них ЛОС эвкалипта прутовидного. Для ЛОС эвкалипта прутовидного использовали только HayeSepN, MN 202 и Porapak Q, поскольку при их использовании для ТФМЭ воспроизводится характерный хроматографический профиль. В литературе описаны исследования, демонстрирующие удовлетворительные результаты при хранении аналитов на сорбционных трубках до 25 месяцев [36]. Для установления срока хранения сорбционных микротрубок с ЛОС эвкалипта прутовидного был проведен контроль содержания $\alpha$-пинена, $n$-цимена, 1,8-цинеола, с периодичностью 3 месяца в течение 1 года (табл. 5). Изменение концентрации $\alpha$-пинена, $n$-цимена, 1,8-цинеола находится в пределах $5-10 \%$, что укладывается в величину ОСКО определения концентрации данных компонентов при ТФМЭ, и как следствие - воспроизводится хроматографический спектр листьев эвкалипта прутовидного.

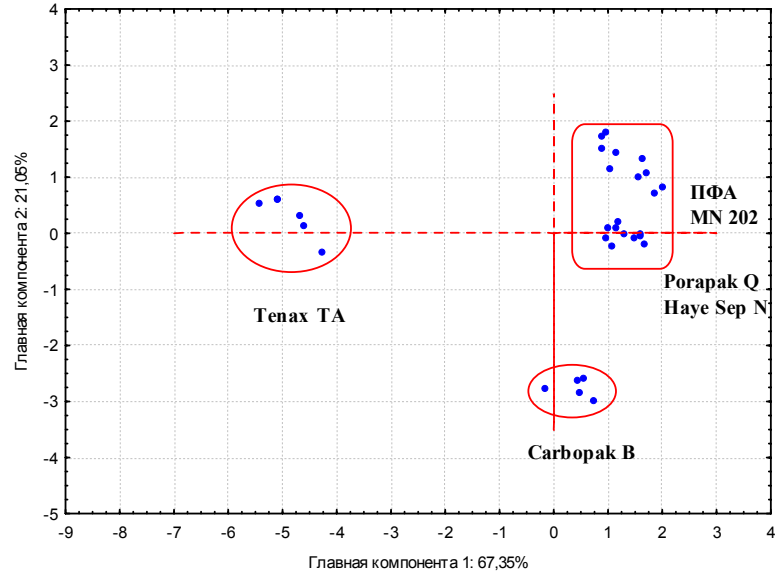

Рис. 7. График в координатах главных компонент 1 и 2 результатов ТФМЭ и ПФА газового экстракта эвкалипта прутовидного (ПФА)

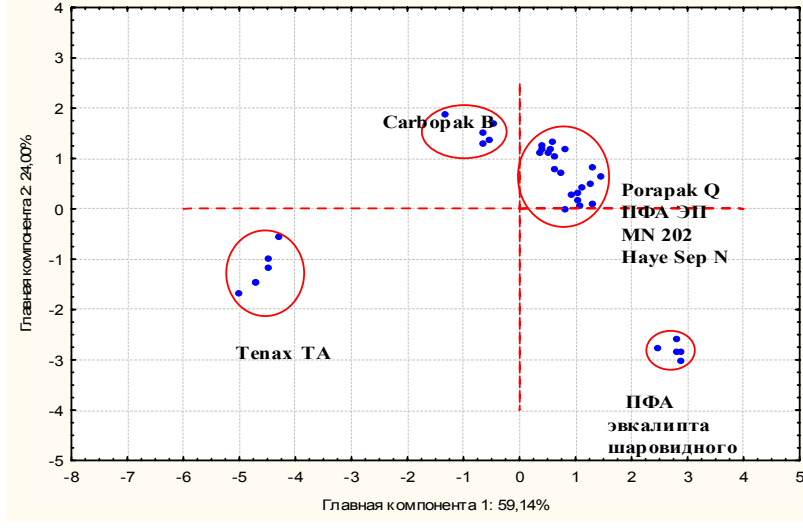

Рис. 8. График в координатах главных компонент 1 и 2 результатов ТФМЭ и ПФА газового экстракта эвкалипта прутовидного (ПФА ЭП) и ПФА эвкалипта шаровидного 
Таблица 5. Оценка срока хранения сорбционных микротрубок с аналитами

\begin{tabular}{|c|c|c|c|c|c|}
\hline \multirow{3}{*}{ Вид ЛОС } & \multirow{3}{*}{ Вещество } & \multirow{3}{*}{ Сроки десорбции } & \multicolumn{3}{|c|}{ Концентрация компонентов в пробе } \\
\hline & & & \multicolumn{3}{|c|}{ после термодесорбции $\overline{\mathrm{x}} \pm \Delta \overline{\mathrm{x}} \pm \Delta, \mathrm{n}=5, \mathrm{P}=0,95$, мкг $/ \mathrm{cm}^{3}$} \\
\hline & & & MN-202 & HayeSep N & Porapak Q \\
\hline \multirow{9}{*}{ 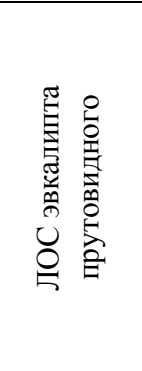 } & & Сразу после ТФМЭ & $1,1 \pm 0,1$ & $0,9 \pm 0,04$ & $2,1 \pm 0,1$ \\
\hline & $\alpha$-пинен & Через 6 месяцев & $1,0 \pm 0,1$ & $0,88 \pm 0,03$ & $2,1 \pm 0,1$ \\
\hline & & Через 12 месяцев & $0,9 \pm 0,1$ & $0,89 \pm 0,03$ & $1,9 \pm 0,1$ \\
\hline & & Сразу после ТФМЭ & $0,4 \pm 0,03$ & $0,42 \pm 0,02$ & $0,41 \pm 0,02$ \\
\hline & $n$-цимен & Через 6 месяцев & $0,35 \pm 0,02$ & $0,40 \pm 0,02$ & $0,39 \pm 0,02$ \\
\hline & & Через 12 месяцев & $0,37 \pm 0,02$ & $0,39 \pm 0,03$ & $0,40 \pm 0,02$ \\
\hline & & Сразу после ТФМЭ & $2,3 \pm 0,1$ & $2,9 \pm 0,1$ & $4,0 \pm 0,1$ \\
\hline & 1,8-цинеол & Через 6 месяцев & $2,2 \pm 0,1$ & $2,7 \pm 0,1$ & $3,9 \pm 0,1$ \\
\hline & & Через 12 месяцев & $2,2 \pm 0,1$ & $2,6 \pm 0,1$ & $3,9 \pm 0,1$ \\
\hline
\end{tabular}

\section{Заключение}

В результате исследования установлено, что хроматографический спектр листьев эвкалипта прутовидного, полученный методом ПФА-ГХ-МС и ПФА-ТФМЭ-ГХ-МС, является воспроизводимой характеристикой, которая может использоваться для идентификации данного растения. Для применения сорбционных трубок в качестве образцов состава ЛОС листьев эвкалипта прутовидного рекомендуется использовать в качестве сорбентов Porapak Q, Наye Sep N, MN 202, которые могут быть взаимозаменяемы. Объем газового экстракта для ТФМЭ составляет $3 \mathrm{~cm}^{3}$. На данный момент срок хранения сорбционных трубок с ЛОС листьев эвкалипта прутовидного составляет 12 месяцев.

\section{Список литературы}

1. Золотов Ю.А. О некоторых тенденциях развития аналитической химии // Заводская лаборатория. Диагностика материалов. 2012. Т. 78, №9. С. 5.

2. Виттенберг А.Г., Иоффе Б.В. Газовая экстракция в хроматографическом анализе: парофазный анализ и родственные методы. Л., 1982. 280 с.

3. Nikam P.H., Kareparamban J., Jadhav A., Kadam V. Future Trends in Standardization of Herbal Drugs // Journal of Applied Pharmaceutical Science. 2012. Vol. 2 (6). Pp. 38-44.

4. Guidance for Industry Botanical Drug Products. U.S. Department of Health and Human Services Food and Drug Administration. Center for Drug Evaluation and Research (CDER) 2004. 48 p.

5. Вигдергауз М.С., Семенченко Л.В., Езрец В.А., Богословский Ю.Н. Качественный газохроматографический анализ. М., 1978. 44 с.

6. Патент №2341792 (РФ). Фингерпринтинг сложных смесей, содержащих углеводороды / Эйде Ингвар, Сулсен Колбьорн / 2008.

7. Фомина Н.В. Идентификация нефтей Самарской области по многомерному массиву данных : автореф. дис. ... канд. хим. наук. Самара, 2014. 25 с.

8. Tistaert C., Dejaegher B., Vander Heyden Y. Chromatographic separation techniques and data handling methods for herbal fingerprints: A review // Analytica Chimica Acta. 2011. Vol. 690. Pp. 148-161.

9. Feng Gan, Runyi Ye. New approach on similarity analysis of chromatographic fingerprint of herbal medicine // Journal of Chromatography A. 2006. Vol. 1104. Pp. 100-105.

10. Latit Giri, Harish C Andola, Vijay Kant Purohit, Rawat M.S.M., Rawal R.S., Bhatt I.D. Chromatographic and spectral fingerprinting standartization of traditional medicines: an overview as modern tools // Research Journal of Phitochemistry. 2010. Vol. 4 (4). Pp. 234-241.

11. Tripathi I.P., Mahendra K.R., Pardhi Yogesh, Dwivedi Atul, Dwivedi Noopa, Kamal Arti, Gupta Priyanka. HPLC Analysis of methanolic extract of some medicinal plant leaves of Myrtaceae family. // International Pharmaceutica Sciencia. 2012. Vol. 2, N3. Pp. 49-53.

12. Alaerts G., Matthijs N., Smeyers-Verbeke J., Vander Heyden Y. Chromatographic fingerprint development for herbal extracts: a screening and optimization methodology on monolithic columns. // J Chromatogr A. 2007. Vol. 1172(1). Pp. 1-8.

13. Dejaegher B., Alaerts G., Matthijs N. Methodology to develop liquid chromatographic fingerprints for the quality control of herbal medicines // Acta Chromatographica. 2010. Vol. 22. N2. Pp. 237-258.

14. Se Mi Yu, Eun Kyung Kim, Je Hyun Lee, Kang Ro Lee, Hong J. Development of Fingerprints for Quality Control of Acorus species by Gas Chromatography/Mass Spectrometry // Bull. Korean Chem. Soc. 2011. Vol. 32. N5. Pp. 1547-1553.

15. Siu-Kay Wong, Shu-Ki Tsui, Sik-Yiu Kwan, Xiao-Li Su, Rui-Chao Lin, Li-Ming Tang, Jian-Xing Chen. Establishment of Characteristic Fingerprint Chromatogram for the Identification of Chinese Herbal Medicines // Journal of Food and Drug Analysis. 2004. Vol. 12. N2. Pp. 110-114. 
16. Rzepa J., Sajewicz M., Baj T., Gorcyca P., Wlodarek M., Glowniak K., Hajnos M. W., Kowaslska T. A Comparison of Methodical Approaches to Fingerprinting of the Volatile Fraction from Winter Savory (Satureja Montana) // Chromatography Research Internetional. 2012. Vol. 2012. P. 8.

17. Daszykowski M., Sajewicz M., Rzepa J., Hajnos M., Staszek D., Wojtal Ł., Kowalska T., Waksmundzka-Hajnos M., Walczak B. Comparative Analysis of the Chromatographic Fingerprints of Twenty Different Sage (Salvia L.) Species // Acta Chromatographica 2009. Vol. 21. N4. Pp. 513-530.

18. Онучак Л.А., Арутюнов Ю.И., Куркин В.А., Платонов И.А., Никитченко Н.В. Газохроматографические характеристики летучих веществ в плодах и препаратах расторопши пятнистой (Silybumm arianum L.) // Журнал аналитической химии. 2012. Т. 67. №6. С. 619-624.

19. Vu D.-T., Nicholas P.E., Erikson C.M. Characterization of Volatiles Using Solid-Phase Microextraction // Gas Chromatography-Mass Spectrometry (SPME/GC-MS). Two Customs Applications. U.S. Customs Serv. Lab. Bull. 2000. Vol. 10. Pp. 1-9.

20. Павлова Л.В., Платонов И.А., Новикова Е.А., Никитченко Н.В. Хромато-масс-спектрометрический анализ (Eucalypti viminalis Labill) с использованием различных способов пробоподготовки // Аналитика и контроль. 2013. T. 17, №3. C. 304-313.

21. Макаров Е.В. Проточная твердофазная микроэкстракция как новый способ сорбционной пробоподготовки в газовой хроматографии : автореф. дис. ... канд. хим. наук. СПб., 2004. 22 с.

22. Другов Ю.С., Родин А.А. Пробоподготовка в экологическом анализе. СПб., 2002. 755 с.

23. Пастухов А.В. Физико-химические свойства и структурная подвижность сверхсшитых полистиролов : автореф. дис. ... докт. хим. наук. М., 2008. 52 с.

24. ГОСТ Р ИСО 16017-1-2007. Воздух атмосферный, рабочей зоны и замкнутых помещений. М., 2008. 41 с.

25. Фармакопея СССР. Изд. 11, вып. 2. М., 1989. 216 с.

26. Ботаника. Руководство к практическим занятиям : учебное пособие / под. ред. Е.И. Барабанова, С.Г. Зайчиковой. М., 2012. 304 с.

27. Siu-Kay Wong, Shu-Ki Tsui, Sik-Yiu Kwan, Xiao-Li Su, Rui-Chao Lin, Li-Ming Tang, Jian-Xing Chen. Establishment of characteristic fingerprint chromatogram for the identification of chinese herbal medicines // Journal of Food and Drug Analysis. 2004. Vol. 12. N2. P. 110.

28. Buenoa P.C.P., Juniorb M.G., Bastos J.K. GC-MS Fingerprinting of 12 Eucalyptus Species // Natural Product Communications 2014. Vol. 9. N12. Pp. 1781-1790.

29. Другов Ю.С., Родин А.А. Газохроматографический анализ природного газа : практическое руководство. М., 2009. $174 \mathrm{c}$.

Поступило в редакичию 12 мая 2016 г.

После переработки 19 мая 2016 г.

Pavlova L.V.*, Platonov I.A., Nikitchenko N.V., Kolesnichenko I.N. APPLICATION OF HEADSPACE ANALYSIS FOR ACQUISITION OF EUCALYPTUS VIMINALIS (EUCALYPTI VIMINALIS LABILL) «FINGERPTINT»

Samara National Research University, Moskovskoye sh.,34, Samara, 443086 (Russia), e-mail: lora-pavlova@mail.ru

The ratio of gas leaf extract component peak areas Eucalyptus (Eucalyptus viminalis Labill): $\alpha$-pinene, camphene, $\beta$ pinene, $\alpha$-fellandren, $p$-cymene, 1,8-cineol, 4-terpineol, $\alpha$-terpineol and aromadendrene was taken for typical chromatographic spectrum. The evaluation of the accuracy of chromatographic spectra of leaves Eucalyptus obtained by Headspace-GC-MS and Headspace -SPME-GC-MS was carried out. For the first time it was invited to use the chromatographic spectra as the objective characteristics of the chemical identity of leaves Eucalyptus instead botanical identification methods. To evaluate the relationship between the results of samples processed by principal component analysis was used program «Statistica». The analysis of the sorption properties of the sorbents Tenax TM TA, Carbopack In, Porapak Q, Haye Sep N, MN-202 with respect to volatile organic compounds (VOCs), eucalyptus leaves was carried out. It was provided to use sorption tubes based on injecting needles, filled selected polymeric sorbents Porapak Q, Haye Sep N, MN-202, on which the concentrated analytes as standard of composition volatile organic compounds (VOCs) Eucalyptus viminalis, including the identification of the raw material. The parameters of the sorption of VOCs and storage life of sorption tubes with analytes ware determined. It is recommended to use the chromatographic spectrum to create a database of chromatographic spectra characteristic of plant raw materials.

Keywords: headspace analysis, chromatographic spectrum, "fingerprint", the overall image of the object, identification, eucalyptus viminalis.

\footnotetext{
* Corresponding author.
} 


\section{References}

1. Zolotov Iu.A. Zavodskaia laboratoriia. Diagnosti-ka materialov, 2012, vol. 78, no. 9, p. 5. (in Russ.).

2. Vittenberg A.G., Ioffe B.V. Gazovaia ekstraktsiia v khromatograficheskom analize: Parofaznyi analiz i rod-stvennye metody. [Gas extraction in the chromatographic analysis: headspace and related methods]. Leningrad, 1982, 280 p. (in Russ.).

3. Nikam P.H., Kareparamban J., Jadhav A., Kadam V. Journal of Applied Pharmaceutical Science, 2012, vol. 2 (6), pp. 38-44.

4. Guidance for Industry Botanical Drug Products. U.S. Department of Health and Human Services Food and Drug Administration. Center for Drug Evaluation and Research (CDER), 2004, 48 p.

5. Vigdergauz M.S., Semenchenko L.V., Ezrets V.A., Bogoslovskii Iu.N. Kachestvennyi gazokhromatograficheskii analiz. [Qualitative GC analysis]. Moscow, 1978, 44 p. (in Russ.).

6. Patent 2341792 (RU). 2008. (in Russ.).

7. Fomina N.V. Identifikatsiia neftei Samarskoi oblasti po mnogomernomu massivu dannykh. Avtoref. dis. ... kand. khim. nauk. [Identification oils Samara region on the multidimensional dataset. Author. Dis. ... Cand. Chem. sciences]. Samara, 2014, 25 p. (in Russ.).

8. Tistaert C., Dejaegher B., Vander Heyden Y. Analytica Chimica Acta, 2011, vol. 690, pp. 148-161.

9. Feng Gan, Runyi Ye. Journal of Chromatography A., 2006, vol. 1104, pp. 100-105.

10. Latit Giri, Harish C Andola, Vijay Kant Purohit, Rawat M.S.M., Rawal R.S., Bhatt I.D. Research Journal of Phitochemistry, 2010, vol. 4 (4), pp. 234-241.

11. Tripathi I.P., Mahendra K.R., Pardhi Yogesh, Dwivedi Atul, Dwivedi Noopa, Kamal Arti, Gupta Priyanka. International Pharmaceutica Sciencia, 2012, vol. 2, no. 3, pp.49-53.

12. Alaerts G., Matthijs N., Smeyers-Verbeke J., Vander Heyden Y. J. Chromatogr. A., 2007, vol. 1172(1), pp. 1-8.

13. Dejaegher B., Alaerts G., Matthijs N. Acta Chromatographica, 2010, vol. 22, no. 2, pp. 237-258.

14. Se Mi Yu, Eun Kyung Kim, Je Hyun Lee, Kang Ro Lee, Hong J. Bull. Korean Chem. Soc., 2011, vol. 32, no. 5, pp. 1547-1553.

15. Siu-Kay Wong, Shu-Ki Tsui, Sik-Yiu Kwan, Xiao-Li Su, Rui-Chao Lin, Li-Ming Tang, Jian-Xing Chen. Journal of Food and Drug Analysis, 2004, vol. 12, no. 2, pp. 110-114.

16. Rzepa J., Sajewicz M., Baj T., Gorcyca P., Wlodarek M., Glowniak K., Hajnos M.W., Kowaslska T. Chromatography Research Internetional, 2012, vol. 2012, p. 8.

17. Daszykowski M., Sajewicz M., Rzepa J., Hajnos M., Staszek D., Wojtal Ł., Kowalska T., Waksmundzka-Hajnos M., Walczak B. Acta Chromatographica, 2009, vol. 21, no. 4, pp. 513-530.

18. Onuchak L.A., Arutiunov Iu.I., Kurkin V.A., Platonov I.A., Nikitchenko N.V. Zhurnal analiticheskoi khimii, 2012, vol. 67, no. 6, pp. 619-624. (in Russ.).

19. Vu D.-T., Nicholas P.E., Erikson C.M. Gas Chromatography-Mass Spectrometry (SPME/GC-MS). Two Customs Applications. U.S. Customs Serv. Lab. Bull., 2000, vol. 10, pp. 1-9.

20. Pavlova L.V., Platonov I.A., Novikova E.A., Nikitchenko N.V. Analitika i kontrol', 2013, vol. 17, no. 3, pp. $304-313$. (in Russ.).

21. Makarov E.V. Protochnaia tverdofaznaia mikroekstraktsiia kak novyi sposob sorbtsionnoi probopodgotovki v gazovoi khromatografii: avtoref. dis. ... kand. khim. nauk. [Flow solid phase microextraction as a new way sorption sample preparation in gas chromatography: Abstract. Dis. ... Cand. Chem. Sciences.]. S.-Peterburg, 2004, 22 p. (in Russ.).

22. Drugov Iu.S., Rodin A.A. Probopodgotovka v ekologicheskom analize, Sankt-Peterburg, 2002, 755 p. (in Russ.).

23. Pastukhov A.V. Fiziko-khimicheskie svoistva i strukturnaia podvizhnost' sverkhsshitykh polistirolov: avtoref. dis. ... dokt. khim. nauk. [Physico-chemical properties and structural mobility hypercrosslinked polystyrene: Abstract. Dis. ... Doctor. Chem. sciences]. Moscow, 2008, 52 p. (in Russ.).

24. GOST R ISO 16017-1-2007. Vozdukh atmosfernyi, rabochei zony i zamknutykh pomeshchenii. [GOST R ISO $16017-$ 1-2007. The air is aspirated, and the work area closed premises]. Moscow, 2008, 41 p. (in Russ.).

25. Farmakopeia SSSR. [Pharmacopoeia of the USSR]. is. 2, Moscow, 1989, 216 p. (in Russ.).

26. Botanika. Rukovodstvo k prakticheskim zaniatiiam: uchebnoe posobie. [Botany. Guide to practical training: a training manual], ed. E.I. Barabanov, S.G. Zaichikova, Moscow, 2012, 304 p. (in Russ.).

27. Siu-Kay Wong, Shu-Ki Tsui, Sik-Yiu Kwan, Xiao-Li Su, Rui-Chao Lin, Li-Ming Tang, Jian-Xing Chen. Journal of Food and Drug Analysis, 2004, vol. 12, no. 2, p. 110.

28. Buenoa P.C.P., Juniorb M.G., Bastos J.K. Natural Product Communications, 2014, vol. 9, no. 12, pp. 1781-1790.

29. Drugov Iu.S., Rodin A.A. Gazokhromatograficheskii analiz prirodnogo gaza: prakticheskoe rukovodstvo. [Gas chromatographic analysis of natural gas: A Practical Guide]. Moscow, 2009, 174 p. (in Russ.).

Received May 12, 2016

Revised May 19, 2016 